\title{
Minkowski-3 Uzayında Timelike Rasyonel Bezier Eğrilerinin Eğrilikleri Üzerine
}

\author{
Hatice KUŞAK SAMANCI ${ }^{*}$ \\ Bitlis Eren Üniversitesi, Matematik Bölümü, Bitlis, Türkiye
}

\begin{abstract}
$\ddot{\mathbf{O z}}$
Bilgisayar destekli geometrik tasarım (BDGT) uygulamaları son yıllarda büyük önem kazanmıştır. Bezier eğrileri BDGT deki önemli bazı eğri türlerindendir. Bezier eğrileri özellikle araba tasarımlarında kullanılmak amacıyla P. Bezier tarafından 1960 yılında keşfedilmiştir. Bu eğriler parametrik yapılarından dolayı tasarımda oldukça kolaylık sağlamaktadırlar. Bezier eğrileri üzerinde şimdiye kadar pek çok çalışma yapılmıştır. Çalışmamızın amacı Minkowski-3 uzayındaki timelike rasyonel Bezier eğrilerinin bazı metrik özelliklerini araştırmaktır. İlk olarak bir timelike rasyonel Bezier eğrisinin Serret Frenet çatısını Minkowski metriğini kullanarak hesapladık. İkinci olarak, timelike rasyonel Bezier eğrilerinin eğriliğini ve burulmasını Minkowski-3 uzayında hesapladık. Daha sonra bu eğrilerin Serret-Frenet çatısının türev formüllerini, timelike eğrilerin Serret-Frenet türev formüllerini kullanarak elde ettik.
\end{abstract}

Anahtar kelimeler: Timelike rasyonel Bezier eğrisi, Minkowski-3 uzayı, eğrilik, burulma.

\section{On Curvatures of The Timelike Rational Bezier Curves in Minkowski 3-Space}

\begin{abstract}
The applications of computer aided geometric design (CAGD) has gained considerable importance in recent years. Bezier curves are some important curve types in CAGD. Bezier curves were discovered in 1960 by P. Bezier, especially for use in automobile design. The curves are very easy to design due to parametric constructions. A lot of studies have been done on Bezier curves so far. The aim of our work is to investigate some metric properties of time-like rational Bezier curves in Minkowski 3-space. Initially we computed the Serret-Frenet frame of a timelike Bezier curve using the Minkowskian metric. Secondly, we calculated the curvature and torsion of the timelike rational Bezier curves in Minkowski 3-space. Later, we obtain the derivative formulas of Serret-Frenet frame of these curves using timelike Serret-Frenet frame.
\end{abstract}

Keywords: Timelike rational Bezier curve, Minkowski 3-space, curvature, torsion.

\section{Giriş}

Bilgisayar Destekli Geometrik Tasarımda (BDGT) eğri üretme teknikleri oldukça önemli bir konudur. Bu eğri üretme tekniklerinden biri olan Bezier eğrileri otomobil tasarımında kullanılmak amaciyla ilk kez 1960 yılında Renault şirketinde mühendis olarak çalışan P. Bezier tarafindan oluşturulmuştur. Bezier eğrileri kontrol noktalarını içeren bir konveks çokgen içerisinde tanımlı Bernstein polinomlarından oluşan parametrik bir eğri çeşididir. Tasarımda kullanım kolaylığı açısından oldukça yaygın bir biçimde tercih edilen Bezier eğrileri içinde bulundukları konveks çokgenin başlangıç ve bitiş noktalarının değiştirilmesi ile eğrinin global olarak değişimini sağlamaktadır. Bu nedenle Bezier eğrisinin uç noktalarındaki teğet ve eğrilik hesapları geometrik açıdan önemlidir. Bezier eğrilerinin geometrik özelliklerine ait temel çalışmalar [1,2] de ayrıntılı bir biçimde verilmiştir. 1992 yılında

\footnotetext{
*Sorumlu yazar: hkusak@beu.edu.tr

Geliş Tarihi: 02.05.2018, Kabul Tarihi: 24.09.2018
} 
Folater n. dereceden Bezier eğrilerinin yüksek mertebeden türevlerini hesaplamıştır [3]. 2004 yılında M. İncesu ve O. Gürsoy Bezier eğrilerinde esas formlar ve eğriliklerle ilgili bir çalışma sunmuştur [4]. 2008 yılına kadar yapılan Bezier eğrileri ile ilgili çalışmalar genellikle Öklid uzayında incelenmiştir. Bezier eğrileri ilk defa 2008 yılında G.H. Georgiev tarafından Minkowski uzayında tanımlanmıştır [5]. B. Pokorná, ve P. Chalmovianski ise kuadratik ve kübik Bezier eğrilerinin Minkowski uzayındaki çizimleri ve sınır koşulları ile ilgili çalışmalar yapmıştır [6, 7]. 2011 yılında H. Ugail ve arkadaşları Bezier yüzeylerini Minkowski uzayında tanımlamıştır [8]. H. Kuşak Samancı ise Minkowski uzayındaki n. dereceden spacelike Bezier ve rasyonel Bezier eğrilerinin uç noktalarındaki Serret-Frenet çatıları, eğrilikleri ve türev formülleri gibi bazı geometrik özellikleri incelemiştir [9, 10]. Minkowski uzayındaki temel kavramlar $[11,12]$ verilmiştir.

Çalışmamızda Minkowski uzayında timelike rasyonel Bezier eğrisi tanımlandıktan sonra bu eğrinin uç noktalarındaki Serret-Frenet çatısı ve eğrilikleri hesaplanmıştır. Bulunan eğrilikler yardımıyla timelike rasyonel Bezier eğrisinin Serret-Frenet çatısına ait türev formülleri hesaplanmıştır.

\section{Materyal ve Metot}

\subsection{Minkowski Uzayı için Temel Bilgiler}

Minkowski uzayında alınan $\eta=\left(\eta_{1}, \eta_{2}, \eta_{3}\right)$ ve $\lambda=\left(\lambda_{1}, \lambda_{2}, \lambda_{3}\right)$ vektörleri için tanımlanan $\mathrm{g}(\eta, \lambda)=-\eta_{1} \lambda_{1}+\eta_{2} \lambda_{2}+\eta_{3} \lambda_{3}$ metriğine Minkowski metriği denir. Minkowski uzayında vektörler Öklid uzayındaki vektörlere göre daha farklı tanımlanmaktadır. $\eta=\left(\eta_{1}, \eta_{2}, \eta_{3}\right) \in \square_{1}^{3}$ vektörü Minkowski 3 uzayında tanımlanan herhangi bir vektör olsun. Eğer $\mathrm{g}(\eta, \eta)>0$ veya $\eta=0$ koşulu sağlanıyorsa $\eta$ vektörüne spacelike vektör denir. $\operatorname{Eğer} g(\eta, \eta)<0$ sağlanıyor ise $\eta$ vektörüne timelike vektör, $\mathrm{g}(\eta, \eta)=0$ ve $\eta \neq 0$ sağlanıyorsa lightlike vektör denir. Minkowski uzayında norm $\|\eta\|_{\mathrm{L}}=\sqrt{|\mathrm{g}(\eta, \eta)|}$ ile tanımlanır. Eğer $\eta$ ve $\lambda$ vektörleri timelike vektörler ise $\phi$ bu iki vektör arasındaki açı olmak üzere Minkowski metriği

$$
g(\eta, \lambda)=-\|\eta\|\|\lambda\| \cosh \phi
$$

biçiminde de yazılabilir. Ayrıca bu iki timelike vektörleri için dış çarpım ve dış çarpımın normu

$$
\begin{aligned}
& \eta \times_{L} \lambda=\left(\eta_{3} \lambda_{2}-\eta_{2} \lambda_{3}, \eta_{1} \lambda_{3}-\eta_{3} \lambda_{1}, \eta_{1} \lambda_{2}-\eta_{2} \lambda_{1}\right), \\
& \left\|\eta \times_{L} \lambda\right\|_{L}=\|\eta\|_{L}\|\lambda\|_{L} \sinh \phi
\end{aligned}
$$

ile tanımlanır [12]. $\alpha$ Minkowski 3 uzayında bir eğri olsun. Eğer her $t$ noktasındaki $\alpha^{\prime}(t)$ teğet vektörü timelike ise $\alpha$ eğrisine timelike eğri denir. Minkowski 3 uzayında bir timelike eğrisi için $\{T, N, B\}$ Serret-Frenet çatısındaki $T$ teğet vektörü timelike vektör, $N$ asal normal vektörü ile $B$ binormal vektörü spacelike vektörleridir. Birim hızlı bir timelike eğrinin Serret-Frenet çatısının vektörleri

$T \times{ }_{L} N=B, N \times_{L} B=-T$ ve $B \times{ }_{L} T=-N$

olmak üzere türev formülleri

$T^{\prime}=\kappa N, N^{\prime}=\kappa T+\tau B, B^{\prime}=-\tau N$,

denklemleri ile verilir [11]. 


\subsection{Rasyonel Bezier Eğrileri için Temel Bilgiler}

Öklid uzayında $\wp_{0}, \wp_{1}, ., \wp_{n} \in \square^{3}$ kontrol noktalarına sahip, $\omega_{i}$ hepsi birden sıfır olmayan skaler ağırlıklı $n$. dereceden rasyonel Bezier ĕgrisi

$$
\wp^{n}(t)=\frac{\sum_{i=0}^{n} \omega_{i} \wp_{i} B_{i, n}(t)}{\sum_{i=0}^{n} \omega_{i} B_{i, n}(t)},
$$

denklemi ile tanımlanır. $t \in[0,1]$ aralı̆̆ı için

$$
B_{i, n}(t)=\left(\begin{array}{c}
\mathrm{n} \\
i
\end{array}\right) t^{\mathrm{i}}(1-t)^{\mathrm{n}-i}
$$

denklemi Bernstein polinomlarını vermektedir. Rasyonel Bezier eğrileri, konik kesitlerin incelenmesinde pratik kullanımı açısından oldukça avantajlı bir eğri üretme tekniğidir. Özellikle quadratik rasyonel Bezier eğrileri olarak adlandırılan ikinci dereceden rasyonel Bezier eğrilerinin ağırlıkları yardımıyla elips, parabol ya da hiperbol olduklarının tespitleri yapılabildiği için rasyonel Bezier eğrilerinin kullanımı oldukça yararlıdır. Eğer $\omega_{1}^{2}-\omega_{0} \omega_{2}<0$ koşulu sağlanıyorsa rasyonel Bezier eğrisi elips belirtir. Eğer $\omega_{1}^{2}-\omega_{0} \omega_{2}=0$ ise parabol ve $\omega_{1}^{2}-\omega_{0} \omega_{2}>0$ ise hiperbol belirtir, [1,2]. Rasyonel Bezier eğrilerinin başlangıç ve bitiş noktalarında konveks çokgene teğettir. Bu nedenle başlangıç ve bitiş noktalarındaki türevlerin hesaplanması önemlidir. M.S. Floater 1991 yılında rasyonel Bezier eğrilerinin oldukça karmaşık bir yapıya sahip olan yüksek mertebeden türevlerini hesaplamıştır. Rasyonel Bezier eğrisinin başlangıç ve bitiş noktalarındaki türevleri

$$
\begin{aligned}
& \left.\frac{d \wp(t)}{d t}\right|_{t=0}=\frac{n \omega_{1}}{\omega_{0}} \Delta \wp_{0} \\
& \left.\frac{d \wp(t)}{d t}\right|_{t=0}=\frac{n \omega_{n-1}}{\omega_{n}} \Delta \wp_{n-1}
\end{aligned}
$$

denklemleri ile vermiştir. Uzun hesaplamalar sonucunda türev vektörlerinin vektörel çarpımları

$$
\begin{aligned}
& \left.\frac{d \wp(t)}{d t}\right|_{t=0} \times\left.\frac{d^{2} \wp(t)}{d t^{2}}\right|_{t=0}=\frac{n^{2}(n-1) \omega_{1} \omega_{2}}{\omega_{0}^{2}}\left(\Delta \wp_{0} \times \Delta \wp_{1}\right) \\
& \left.\frac{d \wp(t)}{d t}\right|_{t=1} \times\left.\frac{d^{2} \wp(t)}{d t^{2}}\right|_{t=1}=\frac{n^{2}(n-1) \omega_{n-1} \omega_{n-2}}{\omega_{n}^{2}}\left(\Delta \wp_{n-1} \times \Delta \wp_{n-2}\right)
\end{aligned}
$$

ve karma çarpımları

$$
\begin{aligned}
& \left.\left(\frac{d \wp(t)}{d t} \frac{d^{2} \wp(t)}{d t^{2}} \frac{d^{3} \wp(t)}{d t^{3}}\right)\right|_{t=0}=\left.n^{3}(n-1)^{2}(n-2) \frac{\omega_{1} \omega_{2} \omega_{3}}{\omega_{0}^{3}}\left(\Delta \wp_{0} \Delta \wp_{1} \Delta \wp_{2}\right)\right|_{t=0} \\
& \left.\left(\frac{d \wp(t)}{d t} \frac{d^{2} \wp(t)}{d t^{2}} \frac{d^{3} \wp(t)}{d t^{3}}\right)\right|_{t=1}=\left.n^{3}(n-1)^{2}(n-2) \frac{\omega_{n-1} \omega_{n-2} \omega_{n-3}}{\omega_{n}^{3}}\left(\Delta \wp_{n-1} \Delta \wp_{n-2} \Delta \wp_{n-3}\right)\right|_{t=1}
\end{aligned}
$$

denklemleri ile hesaplamıştır [3]. 


\section{Bulgular ve Tartışma}

Tanım 3.1. $\square{ }_{1}^{3}$ Minkowski uzayında aynı timelike konide bulunan $\wp_{0}, \wp_{1}, . . \wp_{n}$ kontrol noktaları ve $\omega_{i}$ ağırlıkları ile tanımlı $n$. dereceden bir timelike rasyonel Bezier eğrisi $t \in[0,1]$ için

$$
\wp^{n}(t)=\frac{\sum_{i=0}^{n} \omega_{i} \wp_{i} B_{i, n}(t)}{\sum_{i=0}^{n} \omega_{i} B_{i, n}(t)}
$$

denklemi ile tanımlanır. $\wp_{i} \in \square_{1}^{3}, \mathrm{i}=0,1, ., n$, kontrol noktaları alınarak tanımlanan $\wp^{n}(t)$ timelike rasyonel Bezier eğrisinin $t \in[0,1]$ için birinci mertebeden türevi

$$
\frac{d \wp^{n}(t)}{d t}=\frac{\left[\sum_{i=0}^{n} \omega_{i} \wp_{i} B_{i, n}(t)\right]^{\prime}-\left[\sum_{i=0}^{n} \omega_{i} B_{i, n}(t)\right]^{\prime} \wp^{n}(t)}{\sum_{i=0}^{n} \omega_{i} B_{i, n}(t)}
$$

ile hesaplanır. Timelike rasyonel Bezier eğrisinin $t=0$ başlangıç noktasındaki türevi eğrinin başlangıç noktasındaki teğetini verir ve (6), (7) denklemlerindekine benzer olarak $\wp_{i} \in \square_{1}^{3}, \mathrm{i}=0,1, ., n$, kontrol noktaları için teğeti

$$
\left.\frac{d \wp(t)}{d t}\right|_{t=0}=\frac{n \omega_{1}}{\omega_{0}} \Delta \wp_{0}
$$

eşitliği ile hesaplanır. $\wp_{i} \in \square_{1}^{3}, \mathrm{i}=0,1, ., n$, kontrol noktaları için eğrinin $t=1$ başlangıç noktasındaki teğeti ise

$$
\left.\frac{d \wp(t)}{d t}\right|_{t=0}=\frac{n \omega_{n-1}}{\omega_{n}} \Delta \wp_{n-1}
$$

eşitliği ile hesaplanır. (8), (9), (10), (11) denklemlerinde verilen vektörel ve iç çarpım işlemleri çalışmamızda (1) ve (2) deki Minkowski uzayında tanımlanan vektörel çarpım ve iççarpım işlemi olarak düşünülerek kullanılacaktır.

Teorem 3.2. $\wp^{n}(t)$ rasyonel Bezier eğrisi timelike ise $\wp_{0}$ başlangıç noktasındaki $\Delta \wp_{0}$ teğet vektörü ve $\wp_{n-1}$ bitiş noktasındaki $\Delta \wp_{n-1}$ vektörü timelike vektördür.

İspat: $\wp^{n}(t)$ eğrisi timelike rasyonel Bezier eğrisi ise $\forall t \in[0,1]$ için

$$
g\left(\frac{d \wp^{n}(t)}{d t}, \frac{d \wp^{n}(t)}{d t}\right)<0
$$

koşulu sağlanır. Öyleyse bu koşuldan $\Delta \wp_{0}$ vektörünün timelike vektör olduğu görülmektedir. (6) veya (13) denklemlerindeki $t=0$ başlangıç noktasındaki teğet vektörü (15) denkleminde yerine yazıldığında 
$g\left(\Delta \wp_{0}, \Delta \wp_{0}\right)<0$ sonucu elde edilir. Böylece $\wp_{0}$ başlangıç noktasındaki $\Delta \wp_{0}$ teğet vektörünün timelike vektörü olduğu ispatlanmış olur. Timelike rasyonel Bezier eğrisinin $\wp_{n-1}$ bitiş noktasındaki $\Delta \wp_{n-1}$ teğetinin de benzer yol ile timelike olduğu ispatlanabilir.

Önerme 3.3. $\wp^{n}(t)$ bir rasyonel Bezier eğrisi olsun. Eğer konveks çokgendeki bütün vektörler timelike vektörler ise $\wp^{n}(t)$ rasyonel Bezier eğrisi timelike eğridir.

Önerme 3.4. $\wp_{0}, \wp_{1}, . . \wp_{n} \in R_{1}^{3}$ kontrol noktaları ile tanımlanan $\wp^{n}(t)$ eğrisi bir timelike rasyonel Bezier eğrisi olsun. $\wp^{n}(t)$ eğrisinin birinci mertebeden türevi hodograf olarak adlandırılır. Eğer bütün $\Delta \wp_{i}$ ler aynı konideki timelike vektörler ise bu durumda $\wp^{n}(t)$ hodograf eğrisi aynı konide timelike eğrileridir.

Şimdi $\wp^{n}(t)$ timelike rasyonel Bezier eğrisinin uç noktalarındaki Serret-Frenet çatıları, eğrilikleri, türev formülleri verilecektir.

Düzgün bir $\wp^{n}(t)$ timelike rasyonel Bezier eğrisi birim hızlı olmayıp yay elamanı $\mathrm{s}=\int_{0}^{1}\left\|\frac{d \wp^{\mathrm{n}}}{d t}\right\|_{L} d t$ integrali ile bulunur. $\wp^{n}(t)$ timelike rasyonel Bezier eğrisinin $\mathrm{t}=0$ başlangıç noktasındaki Serret-Frenet çatısı $\left.\{T, N, B\}\right|_{\mathrm{t}=0}$ olsun. Timelike eğrileri için

$$
\begin{array}{lll}
g(T, T)=-1 & g(N, N)=1 & g(B, B)=1, \\
g(T, N)=0 & g(T, B)=0 & g(N, B)=0
\end{array}
$$

özellikleri sağlanacağından Serret-Frenet çatısını oluşturan vektörlerden $T$ teğeti timelike, $N$ asli normali ve $B$ binormali spacelike olduğu görülür. Birim hızlı olmayan $\wp^{n}(t)$ eğrisinin hızı

$$
v=\left\|\frac{d \wp^{n}(t)}{d t}\right\|_{L}=\sqrt{-g\left(\frac{d \wp^{n}(t)}{d t}, \frac{d \wp^{n}(t)}{d t}\right)}
$$

normu ile ve yay uzunluğu parametresi

$$
S=\int_{t_{0}}^{t_{1}} \sqrt{-g\left(\frac{d \wp^{n}(t)}{d t}, \frac{d \wp^{n}(t)}{d t}\right)} d t
$$

integrali ile hesaplanır.

Teorem 3.5. $\wp_{i} \in R_{1}^{3}$ kontrol noktaları ile verilen bir $\wp^{n}(t)$ timelike rasyonel Bezier eğrisi için $\Delta \wp_{i}$ ler aynı konide yer alan vektörler ise $t=0$ noktasındaki $\left.\{T, N, B\}\right|_{t=0}$ Serret-Frenet çatısı

$$
\begin{aligned}
\left.T\right|_{t=0} & =\frac{\Delta \wp_{0}}{\sqrt{-g\left(\Delta \wp_{0}, \Delta \wp_{0}\right)}} \\
\left.N\right|_{t=0} & =\frac{\Delta \wp_{0}}{\left\|\Delta \wp_{0}\right\|_{L}} \operatorname{coth} \theta-\frac{\Delta \wp_{1}}{\left\|\Delta \wp_{1}\right\|_{L}} \operatorname{csch} \theta
\end{aligned}
$$




$$
\left.B\right|_{t=0}=\frac{\Delta \wp_{0} \times_{L} \Delta \wp_{1}}{\left\|\Delta \wp_{0}\right\| \cdot\left\|\Delta \wp_{1}\right\|_{L} \sinh \theta}
$$

denklemleri ile hesaplanır, burada $\theta$ açısı $\Delta \wp_{0}$ ve $\Delta \wp_{1}$ vektörleri arasındaki açıdır.

İspat: $\wp^{n}(t)$ timelike rasyonel Bezier eğrisinin $t=0$ başlangıç noktasındaki $\Delta \wp_{0}$ vektörü timelike olduğu için $\left\|\Delta \wp_{0}\right\|_{L}=\sqrt{-g\left(\Delta \wp_{0}, \Delta \wp_{0}\right)}$ normu ile tanımlıdır. (13) denkleminin kullanılmasıyla pozitif $\omega_{0}, \omega_{1}$ ağıllıkları için başlangıç noktasındaki teğeti

$$
\left.T\right|_{t=0}=\frac{\frac{d \wp^{n}(t)}{d t}}{\left\|\frac{d \wp^{n}(t)}{d t}\right\|_{L}}=\frac{\frac{n \omega_{1}}{\omega_{0}} \Delta \wp_{0}}{\left\|\frac{n \omega_{1}}{\omega_{0}} \Delta \wp_{0}\right\|_{L}}=\frac{\Delta \wp_{0}}{\sqrt{-g\left(\Delta \wp_{0}, \Delta \wp_{0}\right)}}
$$

denklemi ile elde edilir. Birim hızlı olmayan $\wp^{n}(t)$ timelike rasyonel Bezier eğrisinin $\mathrm{t}=0$ başlangıç noktasındaki binormal vektörü ise (8) ve (9) denklemlerinden

$$
\begin{aligned}
& \left.B\right|_{t=0}=\frac{\frac{d \wp^{n}(t)}{d t} \times{ }_{L} \frac{d^{2} \wp^{n}(t)}{d t^{2}}}{\left.\left\|\frac{d \wp^{n}(t)}{d t} \times \times_{L} \frac{d^{2} \wp^{n}(t)}{d t^{2}}\right\|_{L}\right|_{t=0}} \\
& =\frac{\frac{n^{2}(n-1) \omega_{1} \omega_{2}}{\omega_{0}^{2}}\left(\Delta \wp_{0} \times \Delta \wp_{1}\right)}{\left\|\frac{n^{2}(n-1) \omega_{1} \omega_{2}}{\omega_{0}^{2}}\left(\Delta \wp_{0} \times \Delta \wp_{1}\right)\right\|_{L}} \\
& =\frac{\Delta \wp_{0} \times_{L} \Delta \wp_{1}}{\left\|\Delta \wp_{0} \times_{L} \Delta \wp_{1}\right\|_{L}}=\frac{\Delta \wp_{0} \times_{L} \Delta \wp_{1}}{\left\|\Delta \wp_{0}\right\|_{L}\left\|\Delta \wp_{1}\right\|_{L} \sinh \theta}
\end{aligned}
$$

eşitliği ile hesaplanır, burada $\theta$ açısı $\Delta \wp_{0}$ ve $\Delta \wp_{1}$ vektörleri arasındaki açıdır. Birim hızlı olmayan timelike rasyonel eğrisinin birim teğet vektörü $\left.T\right|_{t=0}$ timelike, asli normali ve binormali $\left.N\right|_{t=0}$ and $\left.B\right|_{t=0}$ spacelike vektörlerdir. (2) denkleminde tanımlanan Minkowski uzayındaki vektörel çarpımı ile $\left.N\right|_{t=0}=-\left.B\right|_{t=0} \times\left.{ }_{L} T\right|_{t=0}$ asli normali (4) de verilen denklem ile hesaplanır. $\theta$ açısı $\Delta \wp_{0}$ ve $\Delta \wp_{1}$ vektörleri arasındaki açı olmak üzere asli normalin hesaplanışı aşağıda verilmiş̧tir: 


$$
\begin{aligned}
& \left.N\right|_{t=0}=-\left.B\right|_{t=0} \times\left._{L} T\right|_{t=0} \\
& =-\frac{\left(\Delta \wp_{0} \wedge_{L} \Delta \wp_{1}\right) \times_{L} \Delta \wp_{0}}{\left\|\Delta \wp_{0} \wedge_{L} \Delta \wp_{1}\right\|_{L} \cdot\left\|\Delta \wp_{0}\right\|_{L}} \\
& =-\frac{-g\left(\Delta \wp_{0}, \Delta \wp_{0}\right) \cdot \Delta \wp_{1}+g\left(\Delta \wp_{1}, \Delta \wp_{0}\right) \cdot \Delta \wp_{0}}{\left\|\Delta \wp_{0}\right\|_{L}^{2} \cdot\left\|\Delta \wp_{1}\right\|_{L} \cdot \sinh \theta} \\
& =-\frac{\left\|\Delta \wp_{0}\right\|_{L}^{2} \cdot \Delta \wp_{1}-\left\|\Delta \wp_{1}\right\|_{L} \cdot\left\|\Delta \wp_{0}\right\|_{L} \cosh \theta \cdot \Delta \wp_{0}}{\left\|\Delta \wp_{0}\right\|_{L}^{2} \cdot\left\|\Delta \wp_{1}\right\|_{L} \sinh \theta} \\
& =\frac{\Delta \wp_{0}}{\left\|\Delta \wp_{0}\right\|_{L}} \operatorname{coth} \theta-\frac{\Delta \wp_{1}}{\left\|\Delta \wp_{1}\right\|_{L}} \operatorname{csch} \theta .
\end{aligned}
$$

Teorem 3.6. Minkowski uzayında $\wp_{0}, \wp_{1}, ., \wp_{n} \in \square_{1}^{3}$ kontrol noktalariyla verilen $n$. dereceden $\wp^{n}(t)$ timelike rasyonel Bezier eğrisinin $t=0$ başlangıç noktasındaki eğrilik ve burulması

$$
\left.\kappa\right|_{t=0}=\frac{n-1}{n} \frac{\omega_{0} \omega_{2}}{\omega_{1}^{2}} \frac{\left\|\Delta \wp_{1}\right\|_{L}}{\left\|\Delta \wp_{0}\right\|_{L}^{2}} \cdot \sinh \theta,\left.\quad \tau\right|_{t=0}=-\frac{n-2}{n} \frac{\omega_{0} \omega_{3}}{\omega_{1} \omega_{2}} \frac{\operatorname{det}\left(\Delta \wp_{0}, \Delta \wp_{1}, \Delta \wp_{2}\right)}{\left\|\Delta \wp_{0} \times_{L} \Delta \wp_{1}\right\|_{L}^{2}}
$$

denklemleri ile hesaplanır.

İspat: Minkowski uzayındaki vektörel çarpımın özelliklerinin ve (8) denkleminin kullanılmasıyla $\wp^{n}(t)$ timelike rasyonel Bezier eğrisinin başlangıç noktasındaki eğriliği

$$
\begin{aligned}
& \left.\kappa\right|_{t=0}=\frac{\left\|\frac{d \wp^{n}(t)}{d t} \times \times_{L} \frac{d^{2} \wp^{n}(t)}{d t^{2}}\right\|_{L}}{\left.\left\|\frac{d \wp^{n}(t)}{d t}\right\|_{L}^{3}\right|_{t=0}} \\
& =\frac{\left\|\frac{n^{2}(n-1) \omega_{1} \omega_{2}}{\omega_{0}^{2}}\left(\Delta \wp_{0} \times_{L} \Delta \wp_{1}\right)\right\|_{L}}{\left\|\frac{n \omega_{1}}{\omega_{0}} \Delta \wp_{0}\right\|_{L}^{3}} \\
& =\frac{n-1}{n} \frac{\omega_{0} \omega_{2}}{\omega_{1}^{2}} \frac{\left\|\Delta \wp_{0} \times_{L} \Delta \wp_{1}\right\|_{L}}{\left\|\Delta \wp_{0}\right\|_{L}^{3}}=\frac{n-1}{n} \frac{\left\|\Delta \wp_{0}\right\|_{L} \cdot\left\|\Delta \wp_{1}\right\|_{L} \sinh \theta}{\left\|\Delta \wp_{0}\right\|_{L}^{3}} \\
& =\frac{n-1}{n} \frac{\omega_{0} \omega_{2}}{\omega_{1}^{2}} \frac{\left\|\Delta \wp_{1}\right\|_{L}}{\left\|\Delta \wp_{0}\right\|_{L}^{2}} \cdot \sinh \theta
\end{aligned}
$$

eşitlikleri ile hesaplanır, burada $\theta$ açısı $\Delta \wp_{0}$ ve $\Delta \wp_{1}$ vektörleri arasındaki açıdır. (10) denkleminin kullanılmasıyla $\wp^{n}(t)$ eğrisinin $t=0$ başlangıç noktasındaki burulması için 


$$
\begin{aligned}
\left.\tau\right|_{t=0} & =\left.\frac{\left(\frac{d \wp^{n}(t)}{d t} \frac{d^{2} \wp^{n}(t)}{d t^{2}} \frac{d^{3} \wp^{n}(t)}{d t^{3}}\right)}{\left\|\frac{d \wp^{n}(t)}{d t} \times_{L} \frac{d^{2} \wp^{n}(t)}{d t^{2}}\right\|^{2}}\right|_{t=0} \\
& =\frac{n^{3}(n-1)^{2}(n-2) \frac{\omega_{1} \omega_{2} \omega_{3}}{\omega_{0}^{3}}\left(\Delta \wp_{0} \Delta \wp_{1} \Delta \wp_{2}\right)}{\left\|\frac{n^{2}(n-1) \omega_{1} \omega_{2}}{\omega_{0}^{2}}\left(\Delta \wp_{0} \times_{L} \Delta \wp_{1}\right)\right\|_{L}^{2}} \\
& =\frac{n-2}{n} \frac{\omega_{0} \omega_{3}}{\omega_{1} \omega_{2}} \frac{g\left(\Delta \wp_{0} \times{ }_{L} \Delta \wp_{1}, \Delta \wp_{2}\right)}{\left\|\Delta \wp_{L}\left(\Delta \wp_{1}-\Delta \wp_{0}\right)\right\|_{L}^{2}} \\
= & -\frac{n-2}{n} \frac{\omega_{0} \omega_{3}}{\omega_{1} \omega_{2}} \frac{\operatorname{det}\left(\Delta \wp_{0}, \Delta \wp_{1}, \Delta \wp_{2}\right)}{\left\|\Delta \wp_{0} \times{ }_{L} \Delta \wp_{1}\right\|_{L}^{2}}
\end{aligned}
$$

sonucu elde edilir.

Teorem 3.7. $\Delta \wp_{0}$ ve $\Delta \wp_{1}$ vektörleri arasındaki açı $\theta$ olmak üzere $n$. dereceden $\wp^{n}(t)$ timelike rasyonel Bezier eğrisinin $t=0$ başlangıç noktasındaki Serret-Frenet türev formülleri

$$
\begin{aligned}
\left.\mathbf{T}^{\prime}\right|_{t=0}= & \left.(n-1) \frac{\omega_{2}}{\omega_{1}} \frac{\left\|\Delta \wp_{1}\right\|_{L}}{\left\|\Delta \wp_{0}\right\|_{L}} \cdot \sinh \theta \cdot \mathbf{N}\right|_{t=0} \\
\left.\mathbf{N}^{\prime}\right|_{t=0}= & \left.(n-1) \frac{\omega_{2}}{\omega_{1}} \frac{\left\|\Delta \wp_{1}\right\|_{L}}{\left\|\Delta \wp_{0}\right\|_{L}} \cdot \sinh \theta \mathbf{T}\right|_{t=0} \\
& -\left.(n-2) \frac{\omega_{3}}{\omega_{2}} \frac{\operatorname{det}\left(\Delta \wp_{0}, \Delta \wp_{1}, \Delta \wp_{2}\right)}{\left\|\Delta \wp_{0} \times_{L} \Delta \wp_{1}\right\|_{L}^{2}}\left\|\Delta \wp_{0}\right\|_{L} \cdot \mathbf{B}\right|_{t=0} \\
\left.\mathbf{B}^{\prime}\right|_{t=0}= & \left.(n-2) \frac{\omega_{3}}{\omega_{2}} \frac{\operatorname{det}\left(\Delta \wp_{0}, \Delta \wp_{1}, \Delta \wp_{2}\right)}{\left\|\Delta \wp_{0} \times_{L} \Delta \wp_{1}\right\|_{L}^{2}}\left\|\Delta \wp_{0}\right\|_{L} \cdot \mathbf{N}\right|_{t=0}
\end{aligned}
$$

denklemleri ile verilir.

İspat: $\wp^{n}(t)$ timelike rasyonel Bezier eğrisinin hızı $v_{1}=\left.\left\|\frac{d \wp^{n}(t)}{d t}\right\|_{L}\right|_{t=0}=\frac{n \omega_{1}}{\omega_{0}}\left\|\Delta \wp_{0}\right\|_{L} \quad$ eşitliği ile hesaplanır. Minkowski uzayındaki birim hızlı olmayan timelike eğrileri için tanımlı (5) ile verilen türev denklemlerinin kullanılmasıyla teğet vektörünün türev formülü

$$
\begin{aligned}
\left.\mathbf{T}^{\prime}\right|_{t=0} & =\left.\left.\kappa\right|_{t=0} v_{1} \cdot \mathbf{N}\right|_{t=0} \\
& =\left.\frac{n-1}{n} \frac{\omega_{0} \omega_{2}}{\omega_{1}^{2}} \frac{\left\|\Delta \wp_{1}\right\|_{L}}{\left\|\Delta \wp_{0}\right\|_{L}^{2}} \cdot \sinh \theta \frac{n \omega_{1}}{\omega_{0}}\left\|\Delta \wp_{0}\right\|_{L} \mathbf{N}\right|_{t=0} \\
& =\left.(n-1) \frac{\omega_{2}}{\omega_{1}} \frac{\left\|\Delta \wp_{1}\right\|_{L}}{\left\|\Delta \wp_{0}\right\|_{L}} \cdot \sinh \theta \cdot \mathbf{N}\right|_{t=0}
\end{aligned}
$$


olarak hesaplanır. Asli normalinin türevi

$$
\begin{aligned}
& \left.\mathbf{N}^{\prime}\right|_{t=0}=\left.\kappa v_{1} \mathbf{T}\right|_{t=0}+\left.\tau v_{1} \mathbf{B}\right|_{t=0} \\
& =\left.\frac{n-1}{n} \frac{\omega_{0} \omega_{2}}{\omega_{1}^{2}} \frac{\left\|\Delta \wp_{1}\right\|_{L}}{\left\|\Delta \wp_{0}\right\|_{L}^{2}} \cdot \frac{n \omega_{1}}{\omega_{0}}\left\|\Delta \wp_{0}\right\|_{L} \sinh \theta \mathbf{T}\right|_{t=0} \\
& -\left.\frac{n-2}{n} \frac{\omega_{0} \omega_{3}}{\omega_{1} \omega_{2}} \frac{\operatorname{det}\left(\Delta \wp_{0}, \Delta \wp_{1}, \Delta \wp_{2}\right)}{\left\|\Delta \wp_{0} \times_{L} \Delta \wp_{1}\right\|_{L}^{2}} \frac{n \omega_{1}}{\omega_{0}}\left\|\Delta \wp_{0}\right\|_{L} \cdot \mathbf{B}\right|_{t=0} \\
& =\left.(n-1) \frac{\omega_{2}}{\omega_{1}} \frac{\left\|\Delta \wp_{1}\right\|_{L}}{\left\|\Delta \wp_{0}\right\|_{L}} \cdot \sinh \theta \mathbf{T}\right|_{t=0} \\
& -\left.(n-2) \frac{\omega_{3}}{\omega_{2}} \frac{\operatorname{det}\left(\Delta \wp_{0}, \Delta \wp_{1}, \Delta \wp_{2}\right)}{\left\|\Delta \wp_{0} \times_{L} \Delta \wp_{1}\right\|_{L}^{2}}\left\|\Delta \wp_{0}\right\|_{L} \cdot \mathbf{B}\right|_{t=0}
\end{aligned}
$$

olarak hesaplanır.

$$
\begin{aligned}
\left.\mathbf{B}^{\prime}\right|_{t=0} & =-\left.\tau v_{1} \mathbf{N}\right|_{t=0} \\
& =\left.\frac{n-2}{n} \frac{\omega_{0} \omega_{3}}{\omega_{1} \omega_{2}} \frac{\operatorname{det}\left(\Delta \wp_{0}, \Delta \wp_{1}, \Delta \wp_{2}\right)}{\left\|\Delta \wp_{0} \times_{L} \Delta \wp_{1}\right\|_{L}^{2}} \frac{n \omega_{1}}{\omega_{0}}\left\|\Delta \wp_{0}\right\|_{L} \cdot \mathbf{N}\right|_{t=0} \\
& =\left.(n-2) \frac{\omega_{3}}{\omega_{2}} \frac{\operatorname{det}\left(\Delta \wp_{0}, \Delta \wp_{1}, \Delta \wp_{2}\right)}{\left\|\Delta \wp_{0} \times_{L} \Delta \wp_{1}\right\|_{L}^{2}}\left\|\Delta \wp_{0}\right\|_{L} \cdot \mathbf{N}\right|_{t=0}
\end{aligned}
$$

Şimdi de $\wp^{n}(t)$ timelike rasyonel Bezier eğrisinin $t=1$ bitiş noktasındaki geometrik özelliklerini ispatlayalım:

Teorem 3.8. $\wp^{n}(t)$ timelike rasyonel Bezier eğrisi için $\wp_{i} \in \square_{1}^{3}, \mathrm{i}=0,1, ., n$, kontrol noktaları olsun. $\Delta \wp_{i}$ ler aynı konide yer alan vektörler ise $t=1$ bitiş noktasındaki $\left.\{T, N, B\}\right|_{t=1}$ Serret-Frenet çatısı

$$
\begin{aligned}
\left.T\right|_{t=1} & =\frac{\Delta \wp_{n-1}}{\sqrt{-g\left(\Delta \wp_{n-1}, \Delta \wp_{n-1}\right)}} \\
\left.N\right|_{t=1} & =\frac{\Delta \wp_{n-1}}{\left\|\Delta \wp_{n-1}\right\|_{L}} \operatorname{coth} \varphi-\frac{\Delta \wp_{n-2}}{\left\|\Delta \wp_{n-2}\right\|_{L}} \operatorname{csch} \varphi \\
\left.B\right|_{t=1} & =\frac{\Delta \wp_{n-1} \times_{L} \Delta \wp_{n-2}}{\left\|\Delta \wp_{n-1}\right\| \cdot\left\|\Delta \wp_{n-2}\right\|_{L} \sinh \varphi}
\end{aligned}
$$

denklemleri ile hesaplanır, burada $\varphi$ açısı $\Delta \wp_{n-1}$ ve $\Delta \wp_{n-2}$ vektörleri arasındaki açıdır.

İspat: $\wp^{n}(t)$ timelike rasyonel Bezier eğrisinin $t=1$ bitiş noktasındaki $\Delta \wp_{n-1}$ vektörü timelike olduğu için $\left\|\Delta \wp_{n-1}\right\|_{L}=\sqrt{-g\left(\Delta \wp_{n-1}, \Delta \wp_{n-1}\right)}$ normu ile tanımlıdır. (7) denkleminin kullanılmasıyla timelike rasyonel Bezier eğrisinin $\omega_{n}, \omega_{n-1}$ ağırlıkları için bitiş noktasındaki teğeti 


$$
\left.T\right|_{t=1}=\frac{\frac{d \wp^{n}(t)}{d t}}{\left\|\frac{d \wp^{n}(t)}{d t}\right\|_{L}}=\frac{\frac{n \omega_{n-1}}{\omega_{n}} \Delta \wp_{n-1}}{\left\|\frac{n \omega_{n-1}}{\omega_{n}} \Delta \wp_{n-1}\right\|_{L}}=\frac{\Delta \wp_{n-1}}{\sqrt{-g\left(\Delta \wp_{n-1}, \Delta \wp_{n-1}\right)}}
$$

denklemi ile elde edilir. Birim hızlı olmayan $\wp^{n}(t)$ timelike rasyonel Bezier eğrisinin $t=1$ bitiş noktasındaki binormal vektörü ise (9) denkleminin yerine yazılmasıyla

$$
\begin{aligned}
& \left.B\right|_{t=1}=\left.\frac{\frac{d \wp^{n}(t)}{d t} \times{ }_{L} \frac{d^{2} \wp^{n}(t)}{d t^{2}}}{\left\|\frac{d \wp^{n}(t)}{d t} \times \frac{d^{2} \wp^{n}(t)}{d t^{2}}\right\|_{L}}\right|_{t=1} \\
& =\frac{\frac{n^{2}(n-1) \omega_{n-1} \omega_{n-2}}{\omega_{n}^{2}}\left(\Delta \wp_{n-1} \times \Delta \wp_{n-2}\right)}{\left\|\frac{n^{2}(n-1) \omega_{n-1} \omega_{n-2}}{\omega_{n}^{2}}\left(\Delta \wp_{n-1} \times \Delta \wp_{n-2}\right)\right\|_{L}} \\
& =\frac{\Delta \wp_{n-1} \times_{L} \Delta \wp_{n-2}}{\left\|\Delta \wp_{n-1} \times_{L} \Delta \wp_{n-2}\right\|_{L}}=\frac{\Delta \wp_{n-1} \times_{L} \Delta \wp_{n-2}}{\left\|\Delta \wp_{n-1}\right\|_{L}\left\|\Delta \wp_{n-2}\right\|_{L} \sinh \varphi}
\end{aligned}
$$

eşitliği ile hesaplanır, burada $\varphi$ açısı $\Delta \wp_{n-1}$ ve $\Delta \wp_{n-2}$ vektörleri arasındaki açıdır.

Birim hızlı olmayan timelike rasyonel eğrisinin birim teğet vektörü $\left.T\right|_{t=1}$ timelike, asli normali ve binormali $\left.N\right|_{t=1}$ and $\left.B\right|_{t=1}$ spacelike vektörlerdir. (2) denkleminde tanımlanan Minkowski vektörel çarpımı ile asli normali $\left.N\right|_{t=1}=-\left.B\right|_{t=1} \times\left._{L} T\right|_{t=1}$ denklemi ile hesaplanır. $\varphi$ açısı $\Delta \wp_{n-1}$ ve $\Delta \wp_{n-2}$ vektörleri arasındaki açı olmak üzere asli normalin hesaplanışı aşağıda verilmiştir:

$$
\begin{aligned}
& \left.N\right|_{t=1}=-\left.B\right|_{t=1} \times\left._{L} T\right|_{t=1} \\
& =-\frac{\left(\Delta \wp_{n-1} \wedge_{L} \Delta \wp_{n-2}\right) \times_{L} \Delta \wp_{n-1}}{\left\|\Delta \wp_{n-1} \wedge_{L} \Delta \wp_{n-2}\right\|_{L} \cdot\left\|\Delta \wp_{n-1}\right\|_{L}} \\
& =-\frac{-g\left(\Delta \wp_{n-1}, \Delta \wp_{n-1}\right) \cdot \Delta \wp_{n-2}+g\left(\Delta \wp_{n-2}, \Delta \wp_{n-1}\right) \cdot \Delta \wp_{n-1}}{\left\|\Delta \wp_{n-1}\right\|_{L}^{2} \cdot\left\|\Delta \wp_{n-2}\right\|_{L} \cdot \sinh \varphi} \\
& =-\frac{\left\|\Delta \wp_{n-1}\right\|_{L}^{2} \cdot \Delta \wp_{n-2}-\left\|\Delta \wp_{n-2}\right\|_{L} \cdot\left\|\Delta \wp_{n-1}\right\|_{L} \cosh \varphi \cdot \Delta \wp_{n-1}}{\left\|\Delta \wp_{n-1}\right\|_{L}^{2} \cdot\left\|\Delta \wp_{n-2}\right\|_{L} \sinh \varphi} \\
& =\frac{\Delta \wp_{n-1}}{\left\|\Delta \wp_{n-1}\right\|_{L}} \operatorname{coth} \varphi-\frac{\Delta \wp_{n-2}}{\left\|\Delta \wp_{n-2}\right\|_{L}} \csc h \varphi .
\end{aligned}
$$


Teorem 3.9. Minkowski uzayında $\wp_{0}, \wp_{1}, ., \wp_{n} \in \square_{1}^{3}$ kontrol noktalarıla verilen $n$. dereceden $\wp^{n}(t)$ timelike rasyonel Bezier eğrisinin $t=1$ bitiş noktasındaki eğrilik ve burulması

$\left.\kappa\right|_{t=1}=\frac{n-1}{n} \frac{\omega_{n} \omega_{n-2}}{\omega_{n-1}^{2}} \frac{\left\|\Delta \wp_{n-2}\right\|_{L}}{\left\|\Delta \wp_{n-1}\right\|_{L}^{2}} \cdot \sinh \kappa,\left.\quad \tau\right|_{t=1}=-\frac{n-2}{n} \frac{\omega_{n} \omega_{n-3}}{\omega_{n-1} \omega_{n-2}} \frac{\operatorname{det}\left(\Delta \wp_{n-1}, \Delta \wp_{n-2}, \Delta \wp_{n-3}\right)}{\left\|\Delta \wp_{n} \times_{L} \Delta \wp_{n-2}\right\|_{L}^{2}}$

denklemleri ile hesaplanır.

İspat: Minkowski uzayındaki vektörel çarpımın özelliklerinin kullanılmasıyla $\wp^{n}(t)$ timelike rasyonel Bezier eğrisinin başlangıç noktasındaki eğriliği

$$
\begin{aligned}
& \left.\kappa\right|_{t=1}=\frac{\left\|\frac{d \wp^{n}(t)}{d t} \times \times_{L} \frac{d^{2} \wp^{n}(t)}{d t^{2}}\right\|_{L}}{\left\|\frac{d \wp^{n}(t) \|^{3}}{d t}\right\|_{L}}=\frac{\left\|\frac{n^{2}(n-1) \omega_{n-1} \omega_{n-2}}{\omega_{n}^{2}}\left(\Delta \wp_{n-1} \times_{L} \Delta \wp_{n-2}\right)\right\|_{L}}{\left\|\frac{n \omega_{n-1}}{\omega_{n}} \Delta \wp_{n-1}\right\|_{L}^{3}} \\
& =\frac{n-1}{n} \frac{\omega_{n} \omega_{n-2}}{\omega_{n-1}^{2}} \frac{\left\|\Delta \wp_{n-1} \times_{L} \Delta \wp_{n-2}\right\|_{L}}{\left\|\Delta \wp_{n-1}\right\|_{L}^{3}}=\frac{n-1}{n} \frac{\left\|\Delta \wp_{n-1}\right\|_{L} \cdot\left\|\Delta \wp_{n-2}\right\|_{L} \sinh \varphi}{\left\|\Delta \wp_{n-1}\right\|_{L}^{3}} \\
& =\frac{n-1}{n} \frac{\omega_{n} \omega_{n-2}}{\omega_{n-1}^{2}} \frac{\left\|\Delta \wp_{n-2}\right\|_{L}}{\left\|\Delta \wp_{n-1}\right\|_{L}^{2}} \cdot \sinh \varphi
\end{aligned}
$$

eşitlikleri ile hesaplanır, burada $\varphi$ açısı $\Delta \wp_{n-1}$ ve $\Delta \wp_{n-2}$ vektörleri arasındaki açıdır. (11) denkleminin kullanılmasıyla $\wp^{n}(t)$ eğrisinin $t=1$ bitiş noktasındaki burulması için

$$
\begin{aligned}
\left.\tau\right|_{t=1} & =\left.\frac{\left(\frac{d \wp^{n}(t)}{d t} \frac{d^{2} \wp^{n}(t)}{d t^{2}} \frac{d^{3} \wp^{n}(t)}{d t^{3}}\right)}{\| \frac{d \wp^{n}(t)}{d t} \times\left._{L} \frac{d^{2} \wp^{n}(t)}{d t^{2}}\right|^{2}}\right|_{t=1} \\
& =\frac{n^{3}(n-1)^{2}(n-2) \frac{\omega_{n-1} \omega_{n-2} \omega_{n-3}}{\omega_{n}^{3}}\left(\Delta \wp_{n-1} \Delta \wp_{n-2} \Delta \wp_{n-3}\right)}{\left\|\frac{n^{2}(n-1) \omega_{n-1} \omega_{n-2}}{\omega_{n}^{2}}\left(\Delta \wp_{n-1} \times_{L} \Delta \wp_{n-2}\right)\right\|_{L}^{2}} \\
& =\frac{n-2}{n} \frac{\omega_{n} \omega_{n-3}}{\omega_{n-1} \omega_{n-2}} \frac{g\left(\Delta \wp_{n-1} \times_{L} \Delta \wp_{n-2}, \Delta \wp_{n-3}\right)}{\left\|\Delta \wp_{L}\left(\Delta \wp_{n-2}-\Delta \wp_{n-1}\right)\right\|_{L}^{2}} \\
= & -\frac{n-2}{n} \frac{\omega_{n} \omega_{n-3}}{\omega_{n-1} \omega_{n-2}} \frac{\operatorname{det}\left(\Delta \wp_{n-1}, \Delta \wp_{n-2}, \Delta \wp_{n-3}\right)}{\left\|\Delta \wp_{n-1} \times_{L} \Delta \wp_{n-2}\right\|_{L}^{2}}
\end{aligned}
$$

denklemi elde edilir. 
Teorem 3.10 $\Delta \wp_{n-1}$ ve $\Delta \wp_{n-2}$ vektörleri arasındaki açı $\varphi$ olmak üzere $n$. dereceden $\wp^{n}(t)$ timelike rasyonel Bezier eğrisinin $t=1$ bitiş noktasındaki Serret-Frenet türev formülleri

$$
\begin{aligned}
\left.\mathbf{T}^{\prime}\right|_{t=1}= & \left.(n-1) \frac{\omega_{n-2}}{\omega_{n-1}} \frac{\left\|\Delta \wp_{1}\right\|_{L}}{\left\|\Delta \wp_{0}\right\|_{L}} \cdot \sinh \varphi \cdot \mathbf{N}\right|_{t=1} \\
\left.\mathbf{N}^{\prime}\right|_{t=1}= & \left.(n-1) \frac{\omega_{n-2}}{\omega_{n-1}} \frac{\left\|\Delta \wp_{n-2}\right\|_{L}}{\left\|\Delta \wp_{n-1}\right\|_{L}} \cdot \sinh \varphi \mathbf{T}\right|_{t=1} \\
& -\left.(n-2) \frac{\omega_{n-3}}{\omega_{n-2}} \frac{\operatorname{det}\left(\Delta \wp_{n-1}, \Delta \wp_{n-2}, \Delta \wp_{n-3}\right)}{\left\|\Delta \wp_{n-1} \times_{L} \Delta \wp_{n-2}\right\|_{L}^{2}}\left\|\Delta \wp_{n-1}\right\|_{L} \cdot \mathbf{B}\right|_{t=1} \\
\left.\mathbf{B}^{\prime}\right|_{t=1}= & \left.(n-2) \frac{\omega_{n-3}}{\omega_{n-2}} \frac{\operatorname{det}\left(\Delta \wp_{n-1}, \Delta \wp_{n-2}, \Delta \wp_{n-3}\right)}{\left\|\Delta \wp_{n-1} \times_{L} \Delta \wp_{n-2}\right\|_{L}^{2}}\left\|\Delta \wp_{n-1}\right\|_{L} \cdot \mathbf{N}\right|_{t=1}
\end{aligned}
$$

denklemleri ile verilir.

İspat: $\wp^{n}(t)$ timelike rasyonel Bezier eğrisinin hızı $v_{2}=\left.\left\|\frac{d \wp^{n}(t)}{d t}\right\|_{L}\right|_{t=1}=\frac{n \omega_{n-1}}{\omega_{n}}\left\|\Delta \wp_{n-1}\right\|_{L} \quad$ eşitliği ile hesaplanır. Minkowki uzayındaki birim hızlı olmayan timelike eğrileri için tanımlı (5) denklemi ile teğet vektörünün türev formülü

$$
\begin{aligned}
\left.\mathbf{T}^{\prime}\right|_{t=1} & =\left.\left.\kappa\right|_{t=1} v_{2} \cdot \mathbf{N}\right|_{t=1} \\
& =\left.\frac{n-1}{n} \frac{\omega_{n} \omega_{n-2}}{\omega_{n-1}^{2}} \frac{\left\|\Delta \wp_{n-2}\right\|_{L}}{\left\|\Delta \wp_{n-1}\right\|_{L}^{2}} \cdot \sinh \varphi \frac{n \omega_{n-1}}{\omega_{n}}\left\|\Delta \wp_{n-1}\right\|_{L} \mathbf{N}\right|_{t=1} \\
& =\left.(n-1) \frac{\omega_{n-2}}{\omega_{n-1}} \frac{\left\|\Delta \wp_{n-2}\right\|_{L}}{\left\|\Delta \wp_{n-1}\right\|_{L}} \cdot \sinh \varphi \cdot \mathbf{N}\right|_{t=1}
\end{aligned}
$$

olarak bulunur. Asli normalinin türevi

$$
\begin{aligned}
& \left.\mathbf{N}^{\prime}\right|_{t=1}=\left.\kappa v_{2} \mathbf{T}\right|_{t=1}+\left.\tau v_{2} \mathbf{B}\right|_{t=1} \\
& =\left.\frac{n-1}{n} \frac{\omega_{n} \omega_{n-2}}{\omega_{n-1}^{2}} \frac{\left\|\Delta \wp_{n-2}\right\|_{L}}{\left\|\Delta \wp_{n-1}\right\|_{L}^{2}} \cdot \frac{n \omega_{1}}{\omega_{0}}\left\|\Delta \wp_{n-1}\right\|_{L} \sinh \varphi \mathbf{T}\right|_{t=1} \\
& -\left.\frac{n-2}{n} \frac{\omega_{n} \omega_{n-3}}{\omega_{n-1} \omega_{n-2}} \frac{\operatorname{det}\left(\Delta \wp_{n-1}, \Delta \wp_{n-2}, \Delta \wp_{n-3}\right)}{\left\|\Delta \wp_{n-1} \times_{L} \Delta \wp_{n-2}\right\|_{L}^{2}} \frac{n \omega_{n-1}}{\omega_{n}}\left\|\Delta \wp_{n-1}\right\|_{L} \cdot \mathbf{B}\right|_{t=1} \\
& =\left.(n-1) \frac{\omega_{n-2}}{\omega_{n-1}} \frac{\left\|\Delta \wp_{n-2}\right\|_{L}}{\left\|\Delta \wp_{n-1}\right\|_{L}} \cdot \sinh \varphi \mathbf{T}\right|_{t=1} \\
& -\left.(n-2) \frac{\omega_{n-3}}{\omega_{n-2}} \frac{\operatorname{det}\left(\Delta \wp_{n-1}, \Delta \wp_{n-2}, \Delta \wp_{n-3}\right)}{\left\|\Delta \wp_{n-1} \times_{L} \Delta \wp_{n-2}\right\|_{L}^{2}}\left\|\Delta \wp_{n-1}\right\|_{L} \cdot \mathbf{B}\right|_{t=1}
\end{aligned}
$$

olarak hesaplanır. Binormalinin türevi ise 


$$
\begin{aligned}
\left.\mathbf{B}^{\prime}\right|_{t=1} & =-\left.\tau v_{2} \mathbf{N}\right|_{t=1} \\
& =\left.\frac{n-2}{n} \frac{\omega_{n} \omega_{n-3}}{\omega_{n-1} \omega_{n-2}} \frac{\operatorname{det}\left(\Delta \wp_{n-1}, \Delta \wp_{n-2}, \Delta \wp_{n-3}\right)}{\left\|\Delta \wp_{n-1} \times_{L} \Delta \wp_{n-2}\right\|_{L}^{2}} \frac{n \omega_{n-1}}{\omega_{n}}\left\|\Delta \wp_{n-1}\right\|_{L} \cdot \mathbf{N}\right|_{t=1} \\
& =\left.(n-2) \frac{\omega_{n-3}}{\omega_{n-2}} \frac{\operatorname{det}\left(\Delta \wp_{n-1}, \Delta \wp_{n-2}, \Delta \wp_{n-3}\right)}{\left\|\Delta \wp_{n-1} \times_{L} \Delta \wp_{n-2}\right\|_{L}^{2}}\left\|\Delta \wp_{n-1}\right\|_{L} \cdot \mathbf{N}\right|_{t=1}
\end{aligned}
$$

denklemi ile elde edilir.

\section{Sonuç ve Öneriler}

Rasyonel Bezier eğri yöntemi Bilgisayar Destekli Geometrik Tasarımda önemli bir eğri üretme tekniğidir. Bu çalışmada Minkowski uzayında timelike rasyonel Bezier eğrilerinin geometrik özellikleri ile ilgili bir araştırma yapılmıştır. Özellikle Minkowski uzayının Öklid metriğinden farklı olması sebebiyle timelike rasyonel Bezier eğrilerinin geometrik özelliklerinin de farklı sonuçlandığ görülmektedir.

\section{Kaynaklar}

[1] Farin G. 1996. Curves and Surfaces for Computer-Aided Geometric Design, Academic Press.

[2] Marsh D. 2005. Applied Geometry for Computer Graphics and CAD, Springer-Verlag, Berlin.

[3] Floater M.S. 1992. Derivatives of Rational Bézier Curves. Computer Aided Geometric Design, 9 (3): 161-174.

[4] İncesu M., Gürsoy O. 2004. Bezier Eğrilerinde Esas Formlar ve Eğrilikler, XVII Ulusal Matematik Sempozyumu, Bildiriler Kitab1, Abant İzzet Baysal Üniversitesi, 146-157.

[5] Georgiev G.H. 2008. Spacelike Bezier Curves in the Three-dimensional Minkowski Space, Proceedings of AIP Conference, 1067 (1): 373-380.

[6] Pokorná B., Chalmovianský P. 2011. Quadratic Space-like Bézier Curves in Three Dimensional Minkowski Space, Proceedings of Symposium on Computer Geometry, 20: 104-110.

[7] Pokorná B., Chalmovianský P. 2012. Planar Cubic Space-like Bezier Curves in Three Dimensional Minkowski Space, Proceeding of Syposium on Computer Geometry, 23: 93-98.

[8] Ugail H., Márquez M.C., Y1lmaz A. 2011. On Bézier Surfaces in Three-dimensional Minkowski Space, Computers \& Mathematics with Applications, 62 (8): 2899-2912.

[9] Kuşak Samanc1 H. 2018. Some Geometric Properties of The Spacelike Bezier Curve with a Timelike Principal Normal in Minkowski 3-Space, Cumhuriyet Science Journal, 39 (1): 71-79.

[10] Kuşak Samanc1 H. 2018. Metric Properties of a Spacelike Rational Bezier Curve with a Timelike Principal Normal, Konuralp Journal of Mathematics, 6 (1): 144-148.

[11] Lopez R. 2014. Differential Geometry of Curves and Surfaces in Lorentz-Minkowski Space, International Electronic Journal of Geometry, 7 (1): 44-107.

[12] Ratcliffe, J.G. 2006. Foundations of Hyperbolic Manifolds, Springer Science\&Business Media, LLC, New York, USA, 68-72. 